\title{
Neuronal Activity of the Medulla Oblongata Revealed by Manganese- Enhanced Magnetic Resonance Imaging in a Rat Model of Gastroesophageal Reflux-Related Cough
}

\author{
Zhe CHEN ${ }^{1,2}$, Dachuan GU ${ }^{3}$, Linfeng FAN ${ }^{4}$, Weitao ZHANG ${ }^{5}$, Lejia SUN ${ }^{6}$, Hui CHEN ${ }^{7}$, Rong \\ DONG $^{8}$, Kefang LAI ${ }^{2}$
}

${ }^{1}$ The First People's Hospital of Kunshan, Jiangsu University, Suzhou, China, ${ }^{2}$ State Key Laboratory of Respiratory Disease, The First Affiliated Hospital of Guangzhou Medical University, Guangzhou Institute of Respiratory Disease, Guangzhou, China, ${ }^{3} \mathrm{Fu}$ Wai Hospital, Peking Union Medical College, Beijing, China, ${ }^{4}$ The Second Affiliated Hospital of Zhejiang University School of Medicine, Zhejiang University, Hangzhou, China, ${ }^{5}$ Zhongshan Hospital, Shanghai Medical College, Fudan University, Shanghai, China, ${ }^{6}$ Peking Union Medical College, Beijing, China, ${ }^{7}$ First Affiliated Hospital of Soochow University, Suzhou, China, ${ }^{8}$ Medical School of Southeast University, Nanjing, China

Received November 3, 2017

Accepted August 23, 2018

Epub Ahead of Print October 23, 2018

\section{Summary}

We investigated neuronal activity of the medulla oblongata during gastroesophageal reflux-related cough (GERC). A rat model of GERC was generated by perfusing $\mathrm{HCl}$ into lower esophagus and inducing cough with citric acid. The $\mathrm{HCl}$ group rat was received $\mathrm{HCl}$ perfusion without citric acid-induced cough. The saline control rat was perfused with saline instead and cough was induced. Citric acid-induced cough rat was only induced by citric acid. Blank group rats were fed normally. Fos expressions were observed in medulla oblongata nuclei using immunohistochemistry. Manganese-enhanced magnetic resonance imaging (MEMRI) was performed to detect the $\mathrm{Mn}^{2+}$ signal following intraperitoneal injection of $\mathrm{MnCl}_{2} . \mathrm{HCl}$ perfusion and citric acid-induced cough caused Fos expressions in the nucleus of solitary tract (nTS), dorsal motor nucleus of the vagus (DMV), paratrigeminal nucleus (Pa5), and intermediate reticular nucleus (IRt), which was higher than $\mathrm{HCl}$ group, saline control group, citric acid-induced cough group, and blank group. A high $\mathrm{Mn}^{2+}$ signal was also observed in most of these nuclei in model rats, compared with blank group animals. The $\mathrm{Mn}^{2+}$ signal was also higher in the $\mathrm{HCl}$, saline and citric acid-induced cough group animals, compared with blank group animals. The study showed medulla oblongata neurons were excited in a $\mathrm{HCl}$ perfusion and citric acid-induced cough rat model, and nTS, DMV,
Pa5 and IRt neurons maybe involved in the cough process and signal integrate.

\section{Key words}

Gastroesophageal reflux-related cough - Manganese-enhanced magnetic resonance imaging $\mathrm{c}$-fos $\bullet$ Nucleus of solitary tract • Dorsal motor nucleus of the vagus

\section{Corresponding authors}

K. Lai, State Key Laboratory of Respiratory Disease, The First Affiliated Hospital of Guangzhou Medical University, Guangzhou Institute of Respiratory Disease, 151 Yanjiang Rd., Guangzhou 510120, China. E-mail: klai@163.com and R. Dong, Medical School of Southeast University, 87 Dingjiaqiao, Nanjing 210009, China. E-mail: dongrongshengli@163.com

\section{Introduction}

Chronic cough is the most common symptom of respiratory outpatients, while gastroesophageal reflux (GER) is one of the most common causes of chronic cough (Irwin et al. 1993, Harding and Richter 1997, Lai et al. 2013). Neurons in the medulla oblongata, such as those in the nucleus of the solitary tract (nTS), may 
control cough. However, whether other neurons are activated during gastroesophageal reflux-related cough (GERC) is unclear. Magnetic resonance imaging (MRI) is a new technique and is widely used in neuroscience research. Manganese-enhanced MRI (MEMRI), also called activity-induced manganese-dependent MRI (AIM-MRI), has been employed to study different phenomena in various species. $\mathrm{Mn}^{2+}$ may enter the neurons through calcium $\left(\mathrm{Ca}^{2+}\right)$ channels due to similarities between $\mathrm{Mn}^{2+}$ and $\mathrm{Ca}^{2+}$. More neuronal excitement results in more $\mathrm{Mn}^{2+}$ entry and accumulation, which can be detected using MRI via differences in signal intensity (Aoki et al. 2002, Takeda 2003, Silva et al. 2004). $\mathrm{Mn}^{2+}$ accumulation in medulla oblongata nuclei may reflect neuronal excitation and thus implicate neurons that participate in the process of GERC.

Previous studies have proved that intraesophageal $\mathrm{HCl}$ perfusion could cause airway hyperresponsiveness, airway inflammation and cough in animals (Hamamoto et al. 1997, Kohrogi et al. 2001, Cheng et al. 2014). In this study, a GERC rat model was generated by acid perfusion into the lower esophagus and by inducing cough with citric acid, Neuronal activity was observed via $\mathrm{Mn}^{2+}$ accumulation detected using MRI. We also examined the expression of Fos, a protein marker of neuronal activity in the central nervous system (CNS), and compared the localization of Fos versus $\mathrm{Mn}^{2+}$.

\section{Methods}

\section{Animals and GERC model generation}

Male Sprague-Dawley rats $(\mathrm{n}=60$; body weight 300-350 g; obtained from the Experimental Animal Center of Jiangsu Province) were divided into five groups: model $(\mathrm{HCl}$ perfusion + citric acid-induced cough) group, $\mathrm{HCl}$ ( $\mathrm{HCl}$ perfusion) group, saline (saline perfusion + citric acid-induced cough) group, cough (only citric acid-induced cough) group, and blank group (each group, $\mathrm{n}=12$ ). All animal experimental protocols were approved by Southeast University (permission number 2014062002) and performed in accordance with the guidelines of 'Animal Care and Use' laid down by The Animal Research Committee of Southeast University. According to our previous method (Liu et al. 2013), the model group rats that received acid perfusion were anesthetized with ketamine hydrochloride $(50 \mathrm{mg} / \mathrm{kg}$, intraperitoneally [i.p.]). Then, $0.1 \mathrm{~mol} / \mathrm{l} \mathrm{HCl}$ (including $0.5 \%$ pepsin) was perfused into the lower esophagus ( $8 \mathrm{drops} / \mathrm{min}, 20 \mathrm{~min} / \mathrm{session}$ ) via a stomach tube once a day for 14 consecutive days. Rats in the $\mathrm{HCl}$ group were only perfused with $\mathrm{HCl}$, and without citric acid treatment. Rats in the saline group were perfused with saline. Cough in the model, saline, and cough groups was induced by citric acid treatment $(0.8 \mathrm{~mol} / \mathrm{l})$ for $5 \mathrm{~min}$ once a day for 14 consecutive days. Blank group rats were fed normally. $\mathrm{MnCl}_{2}(0.12 \mathrm{~mol} / \mathrm{l}, 0.45 \mathrm{~g} / \mathrm{kg}$, i.p.) was injected into 6 random rats in each group (including the blank group) on Days 1, 3, 7, 5, 9, 11, and 13 (Fig. 1).

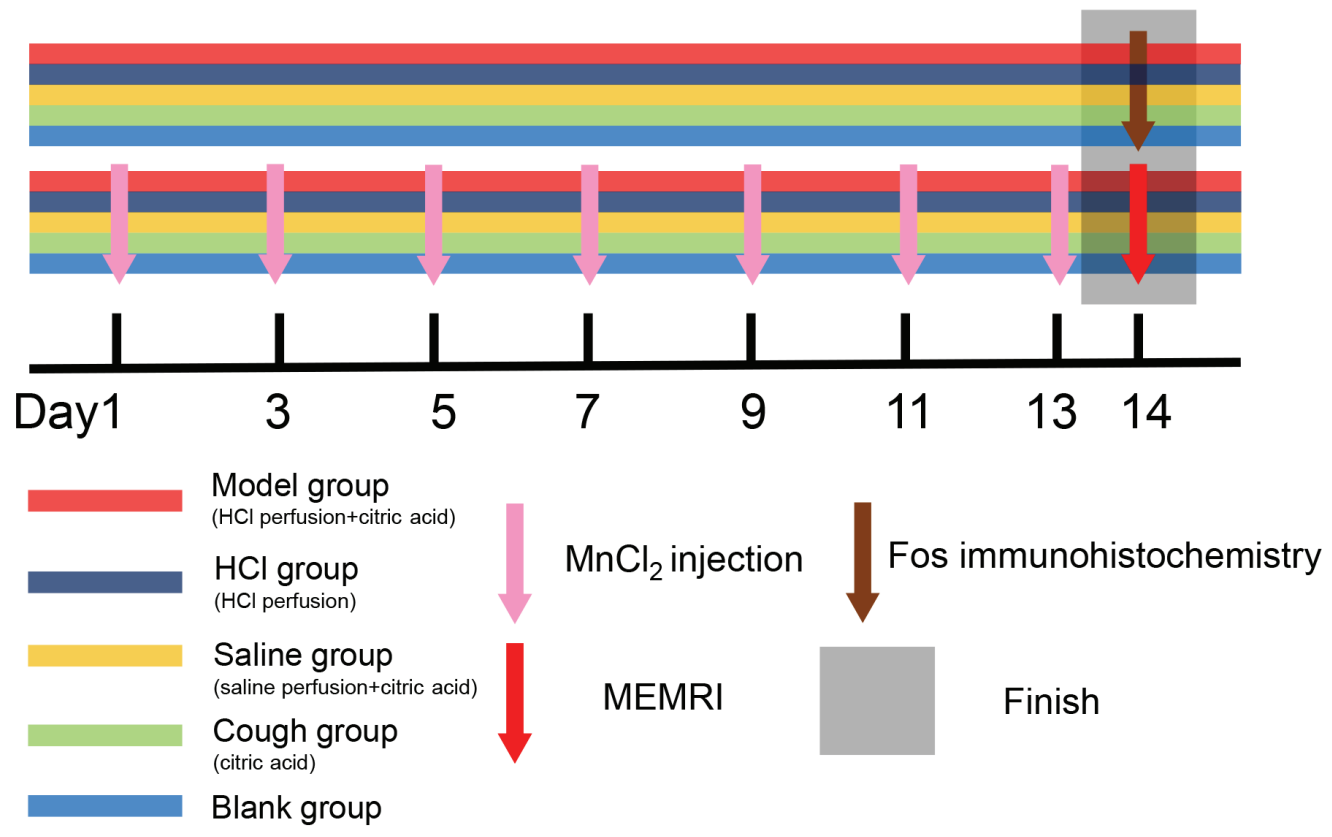

Fig. 1. The experimental procedure. Six animals of each group were for immunohistochemistry and other six animals were for MEMRI. 


\section{Immunohistochemistry}

In the five groups, immunohistochemistry was performed in all rats that were not injected with $\mathrm{MnCl}_{2}$. Animals were deeply anesthetized with urethane $(1 \mathrm{~g} / \mathrm{kg}$, i.p.) and transcardially perfused with $0.3 \%$ phosphate buffered saline (PBS) followed by $4 \%$ paraformaldehyde in PBS. The brainstems were removed, placed in $4 \%$ paraformaldehyde at $4{ }^{\circ} \mathrm{C}$ for $4 \mathrm{~h}$, and then cryoprotected in $30 \%$ sucrose at $4{ }^{\circ} \mathrm{C}$ overnight. Tissues were rapidly frozen with optimal cutting temperature compound and cut into 30- $\mu \mathrm{m}$ thick coronal sections (the total brainstem sections thickness is $2 \mathrm{~mm}$ from rostral and caudal to obex) using a Leica freezing microtome. Brain sections were incubated with $3 \% \mathrm{H}_{2} \mathrm{O}_{2}$ for $15 \mathrm{~min}$ to block endogenous peroxidase activity, washed with $0.3 \%$ PBS ( $3 \times 5 \mathrm{~min})$, incubated for $1 \mathrm{~h}$ at room temperature with a blocking solution (10\% goat serum), and subsequently incubated overnight with a primary antibody (rabbit anti-Fos; 1:500; Santa Cruz). The tissue was washed with $0.3 \%$ PBS $(3 \times 5 \mathrm{~min})$, followed by incubation for $1 \mathrm{~h}$ at room temperature with a biotinylated secondary antibody (goat anti-rabbit; 1:300; Abcam). After washing with $0.3 \%$ PBS $(3 \times 5 \mathrm{~min})$, sections were incubated for $30 \mathrm{~min}$ with avidin/biotinylated horseradish peroxidase (HRP), then washed with $0.3 \%$ PBS $(3 \times 5 \mathrm{~min})$, and reacted with $\mathrm{DAB}$ as a chromogen. Sections were observed using an Olympus light microscope.

\section{MEMRI}

MEMRI was performed using a Bruker 7.0T micro-MR imaging system for rat obex scanning (Fig. 2). Animals were anesthetized with $4 \%$ isoflurane; anesthesia was maintained using $1.5 \%$ isofluraneoxygen/nitrogen (30:70) mixed gas while simultaneously monitoring heart rate and respiratory status. $\mathrm{Mn}^{2+}$ signal intensity changes were detected using rapid acquisition with relaxation enhancement (RARE). T1W anatomical scans were acquired (individual scan time $=10 \min 57 \mathrm{~s}$ $780 \mathrm{~ms} ; \quad$ TR=571 ms; TE=8.09 ms, FOV $3.00 \mathrm{~cm} \times$ $3.00 \mathrm{~cm}$; matrix $384 \times 384 ; 12$ slices; $1.0 \mathrm{~mm}$ slice thickness; $0.078 \times 0.078 \mathrm{~mm}$ in-plane resolution).
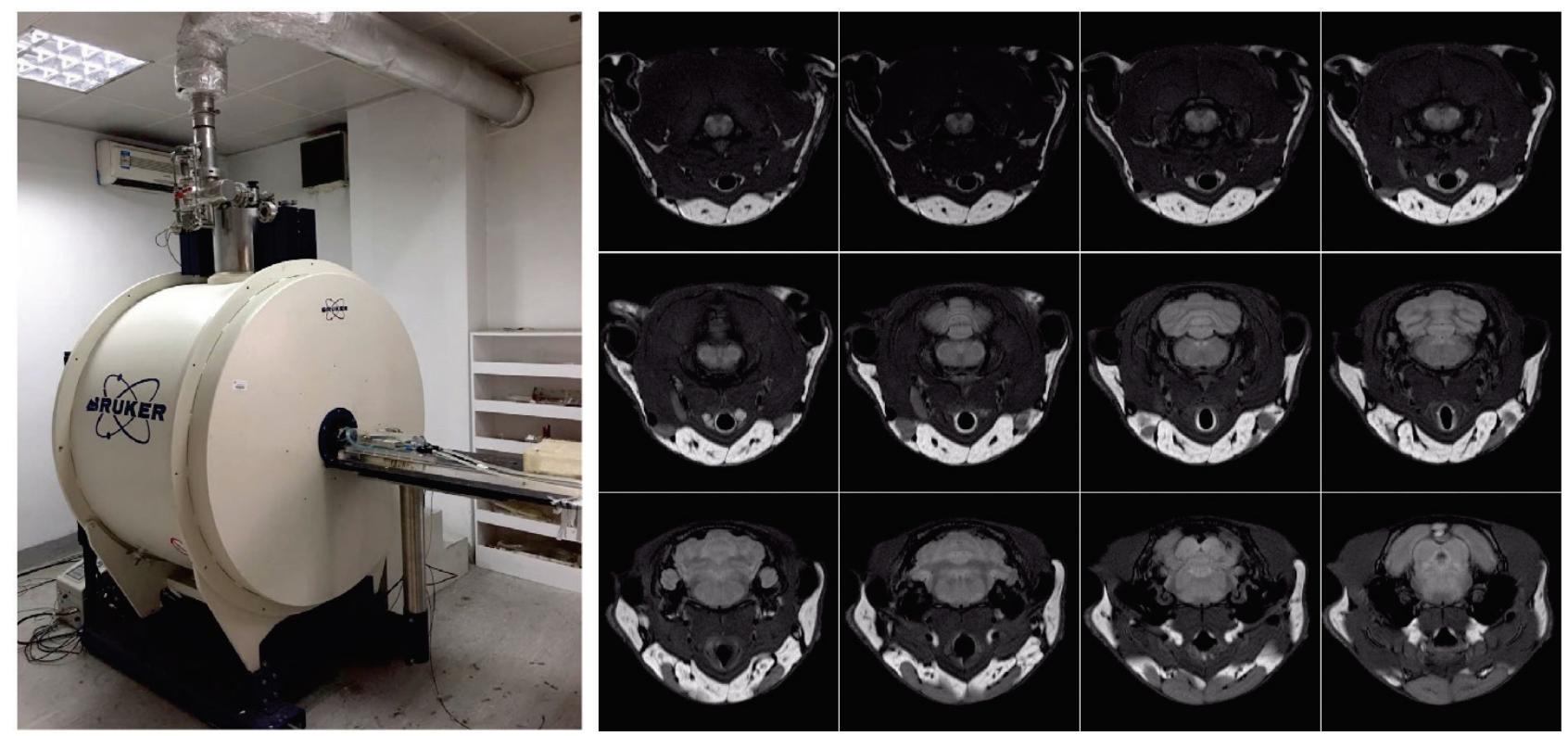

Fig. 2. Bruker 7.0T micro-MR imaging system and rat medulla oblongata obex images.

\section{Statistical analysis}

According to rat brain in stereotaxic coordinates (Paxinos and Watson 2007), Fos-positive neurons stained by immunohistochemistry were observed in the obex nuclei of the medulla oblongata, including nTS, DMV, Pa5, and IRt. Six brain sections were randomly selected in each rat brainstem. Fos-positive neurons were counted using Image-Pro Plus. Paravision 4.0 software was used for MEMRI to measure the regions of interest (ROI) and background noise to calculate a signal-to-noise ratio (SNR). $\mathrm{Mn}^{2+}$ signal changes among the blank group and the other three groups were expressed as a pseudo-color value (pseudo-color value $=$ pixel-value difference $\times$ $0.001)$.

Data were expressed as mean \pm standard deviation $(\bar{x} \pm$ SD). The SPSS 17.0 software was used for 
statistical analysis, including one-way analysis of variance (ANOVA) (comparisons in multiple groups) and paired t test (comparisons between the right and left brain areas in one group). $p<0.05$ was considered statistically significant.

\section{Results}

One rat that received a $\mathrm{MnCl}_{2}$ injection died during the $\mathrm{HCl}$ model preparation.

\section{Fos expression in medulla oblongata nuclei}

Fos-like immunoreactivity (Fos-li) was visualized as brown granules following DAB staining. The greatest Fos-li was observed in the neuronal nuclei (Fig. 3). Fos-positive neurons were mainly distributed in the nTS $(89.31 \pm 9.04)$, dorsal motor nucleus of the vagus (DMV; 61.83 \pm 6.31 ), paratrigeminal nucleus (Pa5; $77.17 \pm 9.01$ ), and intermediate reticular nucleus (IRt; $54.94 \pm 7.59)$ of the model rats $(p<0.05$ compared with each nucleus of the other four groups). Fos-positive

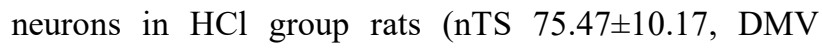
$50.29 \pm 5.27$, Pa5 64.92 \pm 8.83 , IRt 48.26 \pm 6.22$)$ were more than the saline, cough and blank groups $(p<0.05)$. There were no differences in the nuclei observed in the saline and cough groups (nTS, $22.28 \pm 4.44$ vs. 15.58 \pm 3.55 ; DMV, $15.61 \pm 3.86$ vs. $13.14 \pm 2.58 ; \mathrm{Pa} 5,12.19 \pm 2.20$ vs. $14.53 \pm 3.26$; and IRt, $14.94 \pm 3.59$ vs. $15.94 \pm 3.03$; all $p>0.05$ ). Fos-li was rarely observed in the blank group rats. No differences were detected between the right and left side nuclei in the five groups $(p>0.05)$.

\section{Fos expression in model rats}
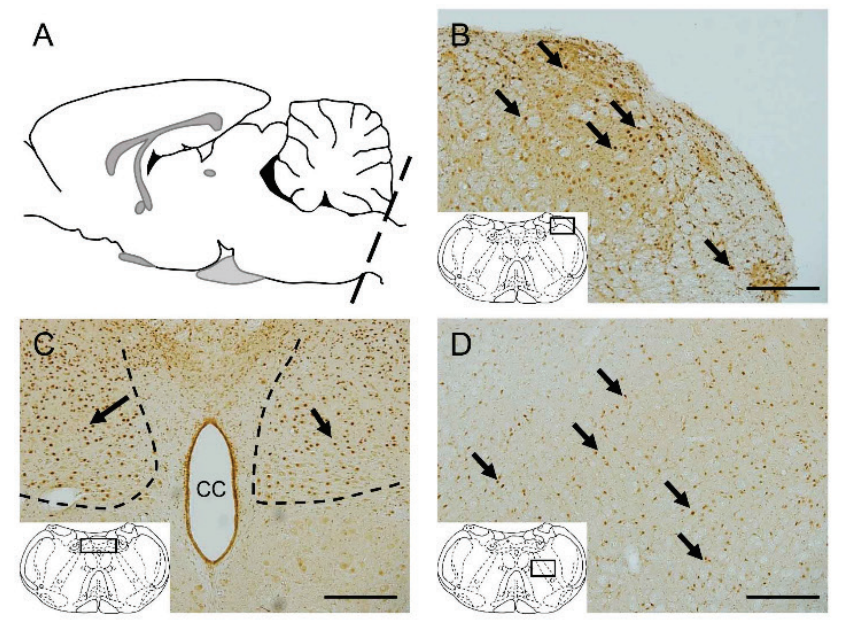

Fos distributions in the nuclei of obex
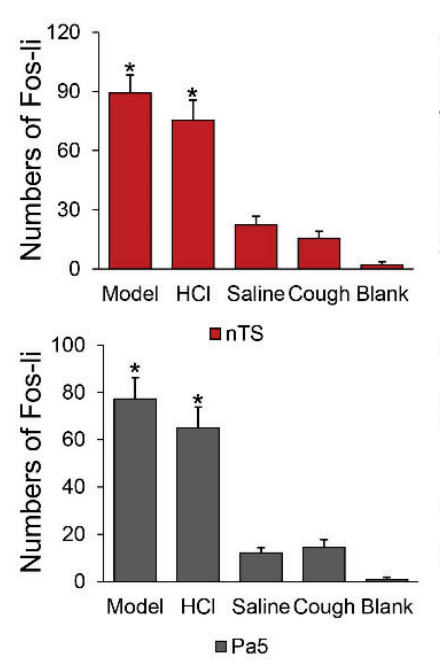

Fig. 3. Fos expression in the model rat and distribution in the nuclei of obex. (A) a frozen brainstem section (black dotted line). (B) Fos expression in Pa5. (C) Fos expression in the DVC (including nTS and DMV). (D) Fos expression in the IRt. The black arrow points Fos-li. Fos-li mainly locating on nTS, DMV, Pa5, and IRt, was more than other four groups. ${ }^{*} p<0.05$. DAB staining. Scale bar $=50 \mu \mathrm{m}$.

\section{$\mathrm{Mn}^{2+}$ signal changes in medulla oblongata nuclei}

$\mathrm{The}^{2+} \mathrm{Mn}^{2+}$ signal was shown in the nTS, DMV, Pa5, and IRt (Fig. 4). In the model group rats, the $\mathrm{Mn}^{2+}$ signal in the nTS was much higher than that observed in the other four groups $(p<0.05)$. The $\mathrm{Mn}^{2+}$ signal was similar between the right and left nTS in all five groups $(p>0.05)$. The DMV of the model group had a higher $\mathrm{Mn}^{2+}$ signal than that of the other four groups $(p<0.05)$. However, the signal of the right DMV $(0.58 \pm 0.06)$ was higher than that of the left $(0.23 \pm 0.04$; $p<0.05)$. Similar results were observed in the $\mathrm{Pa} 5$ of the model group compared with the other four groups $(p<0.05)$. However, the signal of the right $\mathrm{Pa} 5$
$(1.63 \pm 0.12)$ was lower than that of the left $(1.92 \pm 0.19)$ $(p<0.05)$. The $\mathrm{Mn}^{2+}$ signal of both the right and left IRt nuclei in saline was higher than those in the model, $\mathrm{HCl}$ and cough groups (all $p<0.05$ ).

\section{Discussion}

Previous studies have suggested that GER-associated cough is mainly related to neurogenic inflammation of airways, micro aspiration, and esophageal-bronchi reflex (Hamamoto et al. 1997, Kohrogi et al. 2001, Kollarik and Brozmanova 2009). The traditional view is that GERC is due to aspiration of 
gastric contents to the larynx and trachea, however, most patients with GERC only showed distal reflux, rather than proximal reflux, and the micro aspiration theory does not explain the mechanism of GERC (Irwin et al. 2000). Due to the common histological origin of the trachea and esophagus, esophageal-bronchi reflex may contribute to GERC by inducing neurogenic inflammation of airways. Previous study has shown that unilateral vagotomy alleviated neurogenic inflammation and neuronal activities (Chen et al. 2017), which suggests that central nervous system may participate in the process of GERC.
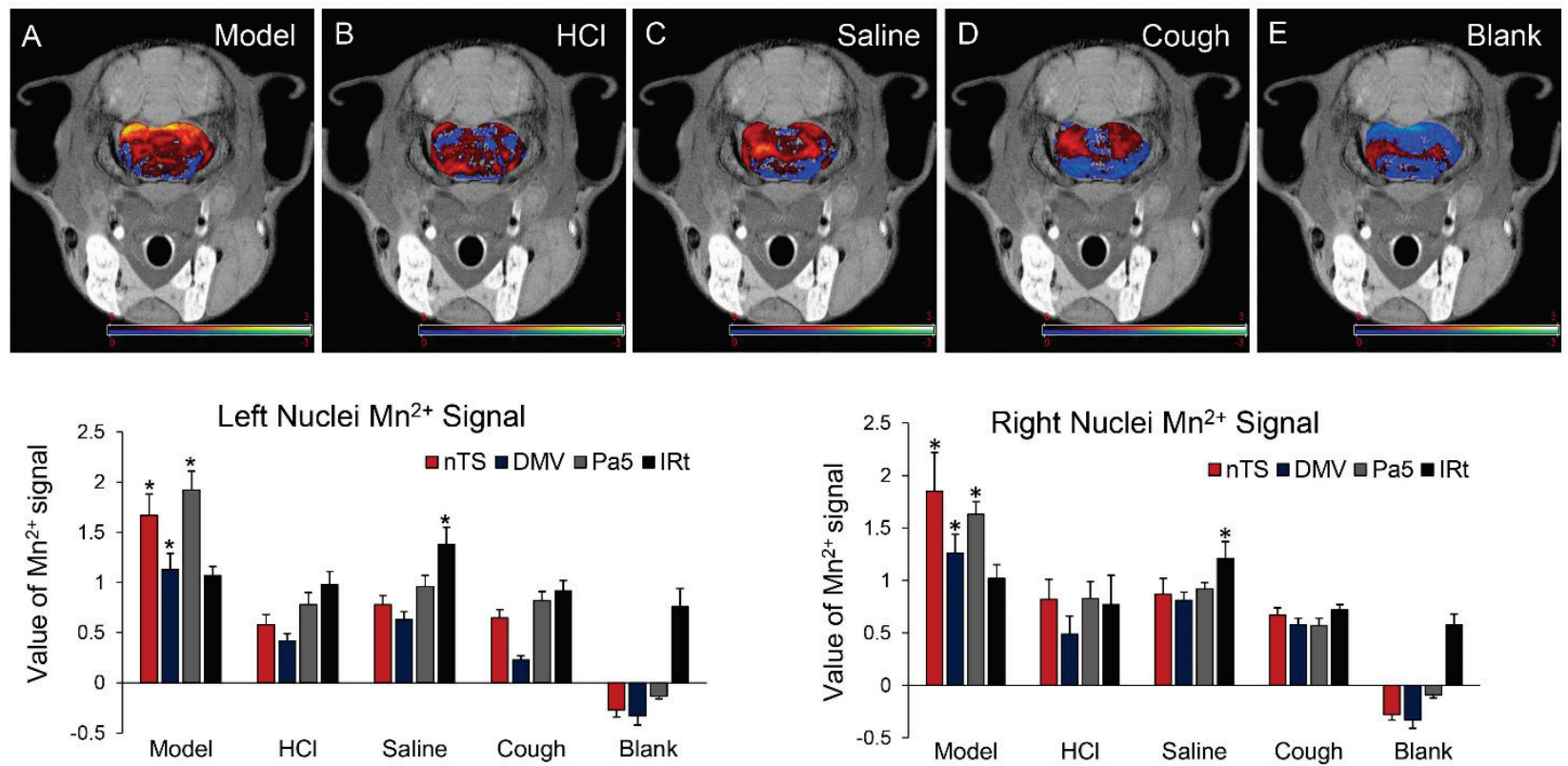

Fig. 4. MEMRI in the rat obex and $\mathrm{Mn}^{2+}$ signal changes in the nuclei. The pictures were (A) model group, (B) $\mathrm{HCl}$ group, (C) saline group, (D) cough group and (E) blank group, respectively. The pseudo-color value was from -3 to 3 . Right and left nuclei $\mathrm{Mn}^{2+}$ signal changes were shown respectively. $* p<0.05$.

$C$-fos can be induced to express Fos protein, a marker of neuronal excitation, in the cell nucleus after stimulation. We included the saline perfusion group and citric acid-induced cough groups to exclude the possibility that surgical tube insertion and liquid perfusion influenced Fos expression. The medulla oblongata is a basal center related to respiration, digestion, and cardiovascular integration. In our study, we found that Fos expression was increased in the model rats, more so than that observed following saline stimulation or cough induced. Previous studies (Gestreau et al. 1997, Ohi et al. 2005, Jakus et al. 2008) have confirmed the location of neurons related to cough using c-fos. Jakus et al. (2008) used Fos to locate the brainstem neurons related to cough, and revealed that a large number of the medulla oblongata, pons, and midbrain neural nuclei are involved in the regulation of coughing in cats. The central terminals of cough receptors are a critical component to cough gating, and by microinjection and dual-tracing studies, terminals, which were localized in the medial subnuclei of NTS, were confirmed (Canning and Mori 2010). Our results indicated that acid perfusion and induced cough resulted in excitation of a greater number of neurons. As we reported previously (Chen et al. 2018), the active neurons in the medulla may participate in the cough and airway inflammation related to the GER.

Cough-related neurons are mainly located in the nTS, a secondary sensory center, which also regulates respiratory functions. Canning and Mori (2010) found that neurons in the cnTS (a subnucleus of the nTS), the location of central cough receptor terminals, were critical components involved in cough gating. Suwanprathes et al. (2003) used Fos to observe neuronal excitation in the brain after a single episode of esophageal acid stimulation.

Fos expression was also observed in the Pa5, another sensory nucleus. The Pa5 receives visceral sensation terminals from the airway and digestive tract via the vagus and glossopharyngeal nerves (Altschuler et al. 1989, Hayakawa et al. 2001, O'Neal and Zheng 2015), and is also referred to as an "extrasolitarial target" 
(Menetrey et al. 1987). McGovern et al. (2015) found dual projecting pathways (Sol airway-specific projections and Pa5 airway-specific projections) from the airway to the brain by virus tracing. These studies indicate that medulla oblongata neurons receive airway and esophageal stimulation signal input, and the signal maybe input to and integrated in higher center (Fig. 5).

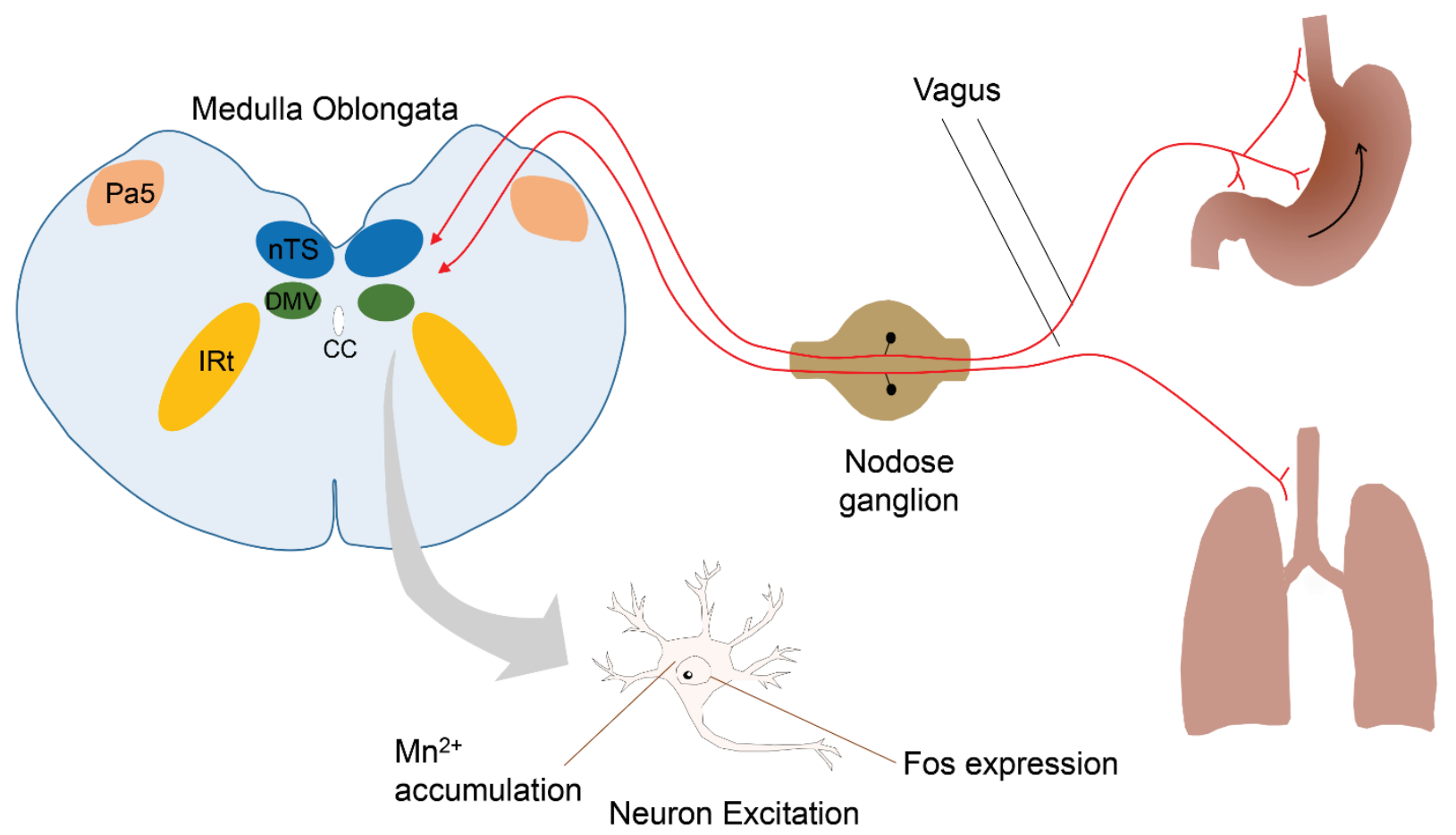

Fig. 5. Neuronal excitation following afferent signals from the periphery. Sensory nuclei of the medulla oblongata receive stimulatory input via the vagal nerves, thereby activating the neurons, which then express Fos protein. $\mathrm{Mn}^{2+}$ also enters into the activated neurons.

The nTS has fiber communications with the DMV and area postrema (AP), and thus is called the dorsal vagal complex (DVC). The DMV directly receives vagal sensory fiber projections, and innervates the airway and digestive tract via efferent fibers. The DVC, together with the IRt, nucleus ambiguus, and ventrolateral medulla, form the medullary visceral zone (MVZ). The MVZ plays a key role in visceral functions. In our study, most of the aforementioned nuclei were excited, particularly following dual stimulation (i.e. $\mathrm{HCl}$ perfusion into the lower esophagus and inhaled citric acid).

Similar results were observed in the MEMRI study. MEMRI was used to confirm the locations of excited neurons in addition to Fos expression. $\mathrm{Mn}^{2+}$ quickly enters into neurons and is released slowly, reflecting neuronal excitation over a period of time; in contrast Fos expression is time restricted. In this study, $\mathrm{Mn}^{2+}$ signals were increased in the nuclei, with higher signals in most of the aforementioned nuclei of the model rats compared with those in the saline and cough groups. The $\mathrm{Mn}^{2+}$ signals in the IRt were not consistent with Fos expression. Fos is an important marker of neuronal activation within the CNS, and also Fos protein expression may be induced by various stimuli. In previous studies reported in the literatures, Fos could be induced in half to one hour after stimuli, and time of peak expression is two hours. Then, Fos expression would be decreased after two hours. The dual stimulation $(\mathrm{HCl}$ and citric acid) in model group would induce more Fos expressions in the nuclei than those in other groups. But $\mathrm{Mn}^{2+}$ differs from Fos protein, it would be accumulated in the cell bodies. Intermediate reticular nucleus (IRt) is a medulla nucleus which is involved in cardiovascular, respiratory, and digest functions. In this study, the cough induced by citric acid (respiratory system) and perfusion stimulation (digest system) would cause more $\mathrm{Mn}^{2+}$ accumulation in the IRt than that in NTS, DMV, and Pa5.

In our study, we have not investigated Fos expression or MEMRI in higher brain areas. Toxic effects are a major drawback of using $\mathrm{Mn}^{2+}$ (Barbeau 1984, Crossgrove and Zheng 2004, Dobson et al. 2004). 
Toxicity, including cardiac, renal, and liver failure, is one of the main limitations to applying this approach in humans. Indeed, MEMRI is now mainly applied in the animal study according to the existing literature, and inappropriate to use in the human clinical study because of the toxic effect of manganese. Instead, blood oxygenation level-dependent functional MRI (BOLDfMRI) is more suitable for human study. Coughing is a complex reflex that involves the CNS and is regarded as a neuropathic disorder (Chung et al. 2013) that may be regulated by circuits involving higher brain areas. Blood oxygenation level-dependent functional MRI (BOLDfMRI) was used to study cough-related mechanisms. Mazzone and colleagues (Mazzone et al. 2007, Mazzone et al. 2011, Farrell et al. 2012, Farrell et al. 2014) found that brain regions, such as the cortex and gyrus cinguli, control the urge to cough, cough suppression, and voluntary cough.

Fos expression and MEMRI showed that medulla oblongata neurons were excited in a $\mathrm{HCl}$ perfusion and citric acid-induced cough rat model, and nTS, DMV, Pa5 and IRt neurons may be involved in the cough process and signal integrate. Medulla oblongata neurons were activated following intra-esophageal $\mathrm{HCl}$ perfusion and inhaled citric acid to induce coughing. These activated neurons may participate in the cough process and cough signal input into higher brain areas. It is also suggested that CNS neurons may be involved in postinfectious cough that responds poorly to standard treatments. For further treatment of GER-associated cough, chronic refractory cough, and even severe asthma, the CNS may serve as a therapeutic target, and blocking the CNS to alleviate airway neurogenic inflammation may provide insight for future drug development.

In conclusion, multiple medulla nuclei were excited in a rat model with $\mathrm{HCl}$ perfusion and citric acidinduced cough, and nTS, DMV, Pa5 and IRt neurons maybe involved in the GERC.

\section{Conflict of Interest}

There is no conflict of interest.

\section{Acknowledgements}

The authors would thank Dr. Ying-ying Bai and Dr. Li-Shan Wang for their help on MRI scanning. This work was supported by Grants 30370621, 30871000 and 81570092 and 81770098 from the National Natural Science Foundation of China, and Grants 2016OP015 and 2007DA780154F0905 from Open Project of State Key Laboratory of Respiratory Disease, and grant KJXW2017071 from the Suzhou Commission of Health and Family Planning, and grant KS18056 of Kunshan Science Project, and Innovative and Entrepreneurial Doctor Project of Jiangsu Province.

\section{References}

ALTSCHULER SM, BAO XM, BIEGER D, HOPKINS DA, MISELIS RR: Viscerotopic representation of the upper alimentary tract in the rat: sensory ganglia and nuclei of the solitary and spinal trigeminal tracts. $J$ Comp Neurol 283: 248-268, 1989.

AOKI I, TANAKA C, TAKEGAMI T, EBISU T, UMEDA M, FUKUNAGA M, FUKUDA K, SILVA AC, KORETSKY AP, NARUSE S: Dynamic activity-induced manganese-dependent contrast magnetic resonance imaging (DAIM MRI). Magn Reson Med 48: 927-933, 2002.

BARBEAU A: Manganese and extrapyramidal disorders (a critical review and tribute to Dr. George C. Cotzias). Neurotoxicology 5: 13-35, 1984.

CANNING BJ, MORI N: An essential component to brainstem cough gating identified in anesthetized guinea pigs. FASEB J 24: 3916-3926, 2010.

CHEN Z, CHEN H, CHEN F, GU D, SUN L, ZHANG W, FAN L, LIN Y, DONG R, LAI K: Vagotomy decreases the neuronal activities of medulla oblongata and alleviates neurogenic inflammation of airways induced by repeated intra-esophageal instillation of $\mathrm{HCl}$ in guinea pigs. Physiol Res 66: 1021-1028, 2017.

CHEN Z, SUN L, CHEN H, GU D, ZHANG W, YANG Z, PENG T, DONG R, LAI K: Dorsal vagal complex modulates neurogenic airway inflammation in a guinea pig model with esophageal perfusion of $\mathrm{HCl}$. Front Physiol 9: 536, 2018.

CHENG YM, CAO AL, ZHENG JP, WANG HW, SUN YS, LIU CF, ZHANG BB, WANG Y, ZHU SL, WU DZ: Airway hyperresponsiveness induced by repeated esophageal infusion of $\mathrm{HCl}$ in guinea pigs. Am J Respir Cell Mol Biol 51: 701-708, 2014. 
CHUNG KF, MCGARVEY L, MAZZONE SB: Chronic cough as a neuropathic disorder. Lancet Respir Med 1: 414-422, 2013.

CROSSGROVE J, ZHENG W: Manganese toxicity upon overexposure. NMR Biomed 17: 544-553, 2004.

DOBSON AW, ERIKSON KM, ASCHNER M: Manganese neurotoxicity. Ann N Y Acad Sci 1012: 115-128, 2004.

FARRELL MJ, COLE LJ, CHIAPOCO D, EGAN GF, MAZZONE SB: Neural correlates coding stimulus level and perception of capsaicin-evoked urge-to-cough in humans. Neuroimage 61: 1324-1335, 2012.

FARRELL MJ, KOCH S, ANDO A, COLE LJ, EGAN GF, MAZZONE SB: Functionally connected brain regions in the network activated during capsaicin inhalation. Hum Brain Mapp 35: 5341-5355, 2014.

GESTREAU C, BIANCHI AL, GRELOT L: Differential brainstem Fos-like immunoreactivity after laryngeal-induced coughing and its reduction by codeine. J Neurosci 17: 9340-9352, 1997.

HAMAMOTO J, KOHROGI H, KAWANO O, IWAGOE H, FUJII K, HIRATA N, ANDO M: Esophageal stimulation by hydrochloric acid causes neurogenic inflammation in the airways in guinea pigs. $J$ Appl Physiol (1985) 82: 738-745, 1997.

HARDING SM, RICHTER JE: The role of gastroesophageal reflux in chronic cough and asthma. Chest 111: 1389-1402, 1997.

HAYAKAWA T, TAKANAGA A, MAEDA S, SEKI M, YAJIMA Y: Subnuclear distribution of afferents from the oral, pharyngeal and laryngeal regions in the nucleus tractus solitarii of the rat: a study using transganglionic transport of cholera toxin. Neurosci Res 39: 221-232, 2001.

IRWIN RS, FRENCH CL, CURLEY FJ, ZAWACKI JK, BENNETT FM: Chronic cough due to gastroesophageal reflux. Clinical, diagnostic, and pathogenetic aspects. Chest 104: 1511-1517, 1993.

IRWIN RS, MADISON JM, FRAIRE AE: The cough reflex and its relation to gastroesophageal reflux. Am J Med $\mathbf{1 0 8}$ (Suppl 4a): 73S-78S, 2000.

JAKUS J, POLIACEK I, HALASOVA E, MURIN P, KNOCIKOVA J, TOMORI Z, BOLSER DC: Brainstem circuitry of tracheal-bronchial cough: c-fos study in anesthetized cats. Respir Physiol Neurobiol 160: 289-300, 2008.

KOHROGI H, HAMAMOTO J, KAWANO O, IWAGOE H, FUJII K, HIRATA N, ANDO M: The role of substance P release in the lung with esophageal acid. Am J Med 111 (Suppl 8A): 25S-30S, 2001.

KOLLARIK M, BROZMANOVA M: Cough and gastroesophageal reflux: insights from animal models. Pulm Pharmacol Ther 22: 130-134, 2009.

LAI K, CHEN R, LIN J, HUANG K, SHEN H, KONG L, ZHOU X, LUO Z, YANG L, WEN F, ZHONG N: A prospective, multicenter survey on causes of chronic cough in China. Chest 143: 613-620, 2013.

LIU C, CHEN R, LUO W, LAI K, ZHONG N: Neurogenic airway inflammation induced by repeated intra-esophageal instillation of $\mathrm{HCl}$ in guinea pigs. Inflammation 36: 493-500, 2013.

MAZZONE SB, COLE LJ, ANDO A, EGAN GF, FARRELL MJ: Investigation of the neural control of cough and cough suppression in humans using functional brain imaging. J Neurosci 31: 2948-2958, 2011.

MAZZONE SB, MCLENNAN L, MCGOVERN AE, EGAN GF, FARRELL MJ: Representation of capsaicin-evoked urge-to-cough in the human brain using functional magnetic resonance imaging. Am J Respir Crit Care Med 176: 327-332, 2007.

MCGOVERN AE, DRIESSEN AK, SIMMONS DG, POWELL J, DAVIS-POYNTER N, FARRELL MJ, MAZZONE SB: Distinct brainstem and forebrain circuits receiving tracheal sensory neuron inputs revealed using a novel conditional anterograde transsynaptic viral tracing system. J Neurosci 35: 7041-7055, 2015.

MENETREY D, LEAH J, DE POMMERY J: Efferent projections of the paratrigeminal nucleus in the rat. Neurosci Lett 73: 48-52, 1987.

O'NEAL SL, ZHENG W: Manganese toxicity upon overexposure: a decade in review. Curr Environ Health Rep 2: 315-328, 2015.

OHI Y, YAMAZAKI H, TAKEDA R, HAJI A: Functional and morphological organization of the nucleus tractus solitarius in the fictive cough reflex of guinea pigs. Neurosci Res 53: 201-209, 2005.

PAXINOS G, WATSON CR: The Rat Brain in Stereotaxic Coordinates (6th Edition). Academic Press, San Diego, 2007, $456 \mathrm{p}$.

SILVA AC, LEE JH, AOKI I, KORETSKY AP: Manganese-enhanced magnetic resonance imaging (MEMRI): methodological and practical considerations. NMR Biomed 17: 532-543, 2004. 
SUWANPRATHES P, NGU M, ING A, HUNT G, SEOW F: c-Fos immunoreactivity in the brain after esophageal acid stimulation. Am J Med 115 (Suppl 3A): 31S-38S, 2003.

TAKEDA A: Manganese action in brain function. Brain Res Brain Res Rev 41: 79-87, 2003. 